\title{
Editorial
}

\section{Advanced Methods to Analyse the Complexity of the Brain}

\author{
Nadia Mammone $\mathbb{D}^{\mathbb{D}},{ }^{1}$ Gaoxiang Ouyang $\mathbb{D}^{2}{ }^{2}$ and Hamed Azami $\mathbb{D}^{3,4}$ \\ ${ }^{1}$ IRCCS Centro Neurolesi Bonino-Pulejo, Messina, Italy \\ ${ }^{2}$ Beijing Normal University, Beijing, China \\ ${ }^{3}$ University of Edinburgh, Edinburgh, UK \\ ${ }^{4}$ Massachusetts General Hospital, Harvard University, Cambridge, MA, USA \\ Correspondence should be addressed to Nadia Mammone; nadia.mammone@unirc.it
}

Received 30 July 2018; Accepted 30 July 2018; Published 8 October 2018

Copyright (c) 2018 Nadia Mammone et al. This is an open access article distributed under the Creative Commons Attribution License, which permits unrestricted use, distribution, and reproduction in any medium, provided the original work is properly cited.

The brain generates bioelectromagnetic fields due to the intra- and extracellular currents associated with the interaction between neurons. Brain activity is also associated with changes in the cellular metabolism and blood flow. Such bioelectromagnetic and metabolic activities can be detected through proper neurophysiological measurements. The spatio-temporal dynamics of the recorded neurophysiological signals are closely related to the age of a subject, to her/ his state of consciousness, to cognitive activities, to the execution of active or passive task (e.g., hyperventilation or external stimulation), to the presence of neurological disorders, to the possible use of medical treatment, and so on. Among the possible neurophysiological acquisition systems, Electroencephalography (EEG), Electrocorticography (ECoG), Magnetoencephalography (MEG), Local Field Potentials (LFP), Event-Related Potential (ERP), Computed Tomography (CT), and functional Magnetic Resonance Imaging (fMRI) are more popular. The data recorded by the abovementioned techniques deeply differ from each other; however, they all are a representation of the output of the complex system that generated them: the brain. The brain is a complex system at least from two perspectives: globally, since it consists of several components dynamically interacting with each other, and locally, as the temporal complexity analysis of univariate time series resulting from a single channel recording can provide information about the local dynamics. Intuitively, complexity in signals or images is associated with meaningful structural richness. In the complexity literature, it is hypothesized that healthy individuals or systems correspond to more complex states due to their ability to adapt to adverse conditions, exhibiting long range correlations, and rich variability at multiple scales, whereas aged and diseased subjects or systems may present complexity alterations, depending on the disease. To quantify the complexity of signals, a number of univariate and multivariate multiscale entropy methods, nonlinear synchronization measures, complex network models, and many others were introduced. This special issue aimed at attracting relevant contributions, both methodological and applications, in the field of complexity analysis of neurophysiological data. It collected valuable contributions in the most varied fields of research ranging from deep brain stimulation (DBS) to brain computer interface (BCI).

In the paper "A Wavelet-Based Correlation Analysis Framework to Study Cerebromuscular Activity in Essential Tremor," the issue of DBS in patients with severe essential tremor (ET) was addressed. Y. Zhao et al. proposed a novel framework to improve the reliability of correlation between LPF signals recorded from the brain and the tremulous electrical activity recorded through Electromyography (EMG). Polygraphic recordings, EEG/LFP/EMG, from two patients that underwent DBS surgery for medically refractory ET were analysed. The authors found a relationship between the thalamic local field potential recordings and the contralateral tremorogenic EMG oscillations at the frequency of the tremor and its first harmonic. Preliminary results endorse the hypothesis that the proposed methodology can identify the thalamic contacts to be selected 
for high-frequency DBS stimulation, with the aim of achieving a better clinical outcome in comparison to the empirical DBS device programming.

In "Automated Epileptic Seizure Detection in Scalp EEG Based on Spatial-temporal Complexity," a novel method is introduced to define a patient-specific detector based on spatial-temporal complexity analysis. The authors evaluated both permutation entropy (PE) and sample entropy (SE). The detectors were tested over a publicly available dataset achieving a $99 \%$ sensitivity by the use of PE. The proposed method also meets the requirements for real-time seizure detection.

In the paper "Automatic Lateralization of Temporal Lobe Epilepsy Based on MEG Network Features Using Support Vector Machines," T. Wu et al., proposed a framework for the lateralization of temporal lobe epilepsy (TLE) using support vector machine (SVM) and weighted network metrics obtained from source-space MEG. It was illustrated that showed that MEG is an appropriate monitoring method for the lateralization of TLE. Additionally, it was found that MEG source space brain-network features can be effectively employed for determining the lateralization.

"Effect of EOG Signal Filtering on the Removal of Ocular Artifacts and EEG-Based Brain-Computer Interface: A Comprehensive Study" is a paper that addresses the issue of ocular artifacts contaminating EEG signals acquired for BCI applications. An optimized low-pass filtering may reduce the effects of electrooculographic (EOG) signals and improve the performance of BCI. The authors found that low-pass filtering at $6-8 \mathrm{~Hz}$ seems to be the optimal filtering range. Furthermore, motor imagery (MI) datasets were used to validate the results. Classification accuracy improved when EOG filtering was applied.

In the article "Using Graph Theory to Assess the Interaction between Cerebral Function, Brain Hemodynamics, and Systemic Variables in Premature Infants," D. Hendrikx et al. showed the ability of graph theory in describing interactions between cerebral function, brain hemodynamics, and systemic variables using some data from a propofol dose finding and pharmacodynamics study in the vulnerable population of preterm infants. The results illustrated that propofol induces a decrease in the signal interaction up to 90 minutes after propofol administration. The clinical recovery phase was mainly determined by the EEG dynamics. Finally, a more pronounced loss in cerebral-systemic interactions with increasing propofol dose was found.

\section{Conflicts of Interest}

The authors declare that they have no conflicts of interest.

Nadia Mammone Gaoxiang Ouyang Hamed Azami 


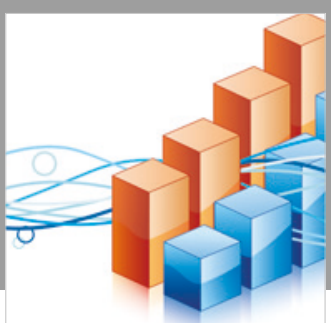

Advances in

Operations Research

\section{-n-m}
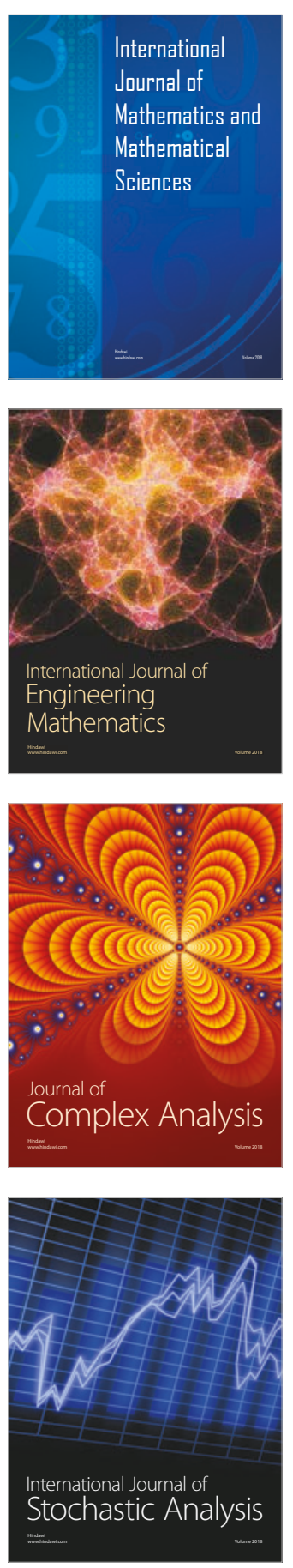
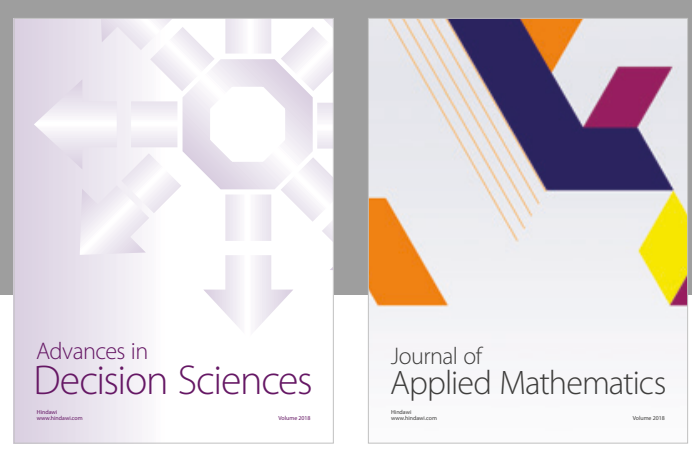

Journal of

Applied Mathematics
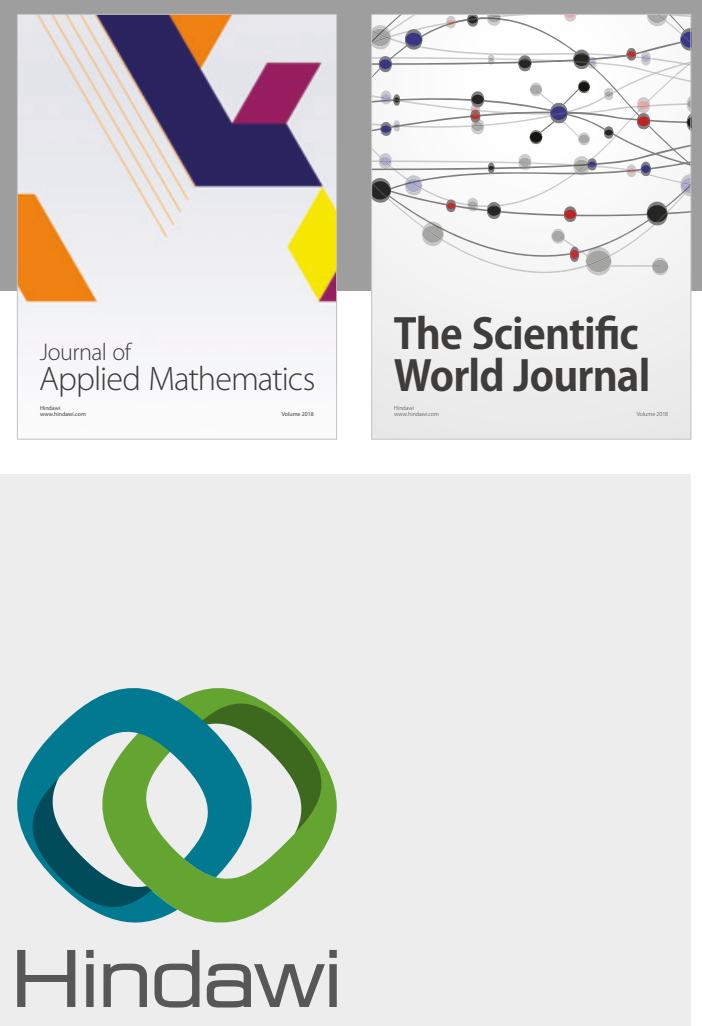

Submit your manuscripts at

www.hindawi.com

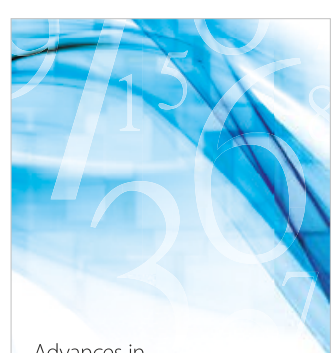

Advances in
Numerical Analysis
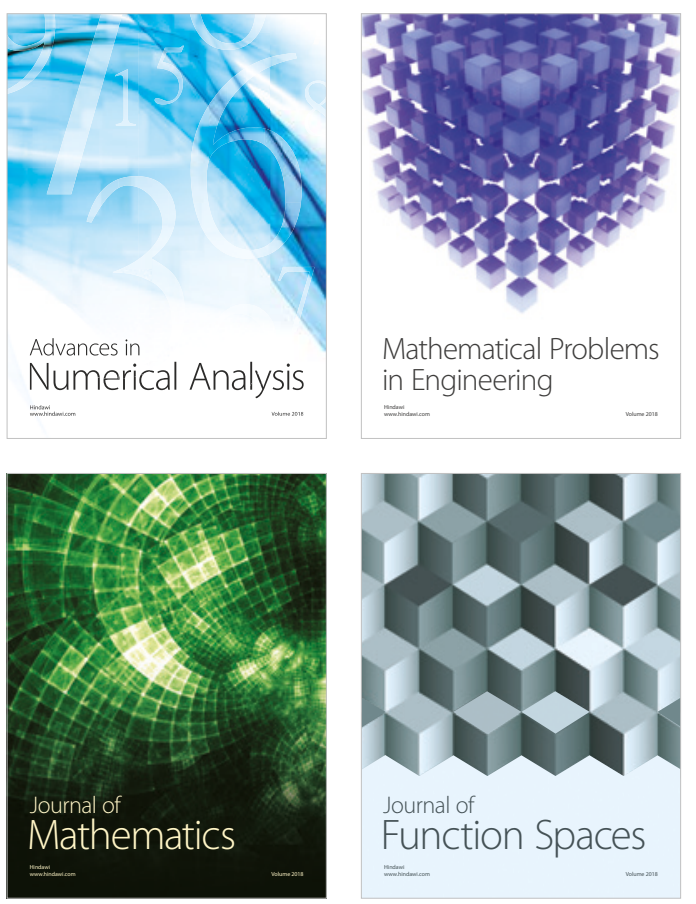

Mathematical Problems in Engineering

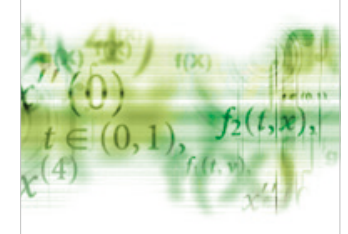

International Journal of

Differential Equations

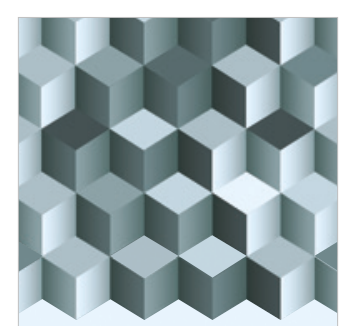

Journal of

Function Spaces
The Scientific

World Journal

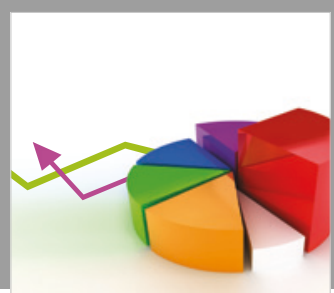

Journal of

Probability and Statistics
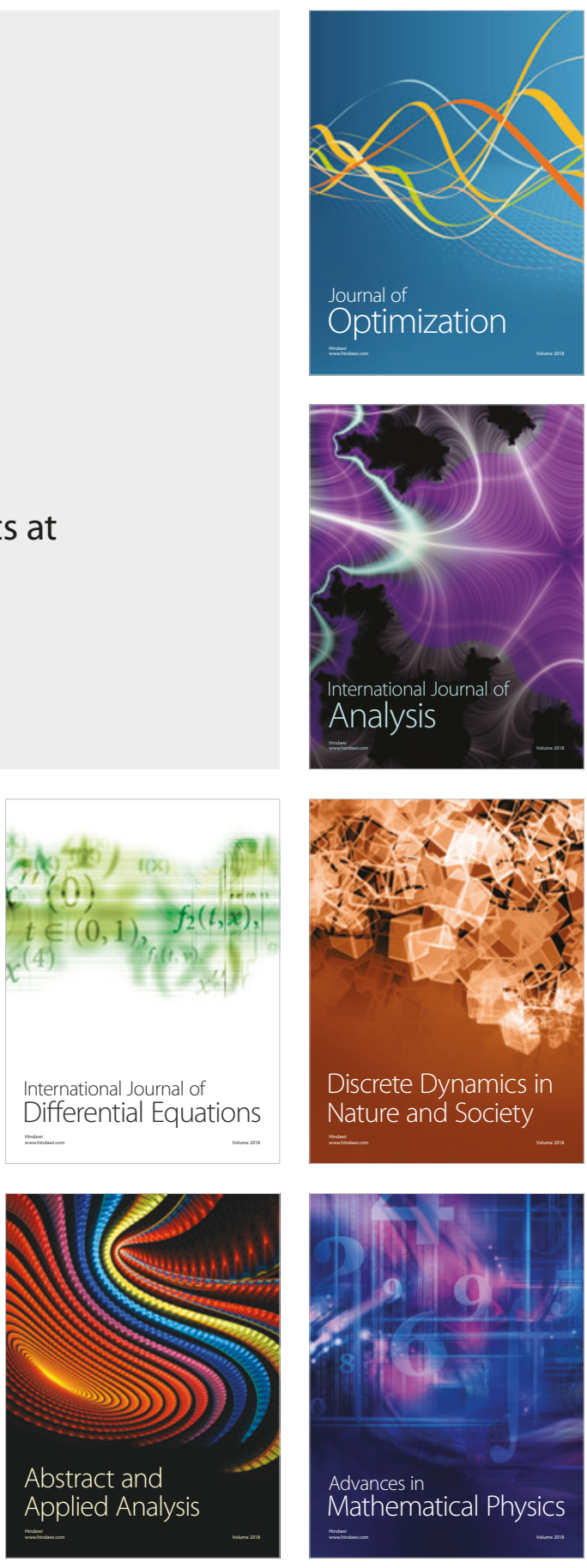